\title{
New Input Data for Synthetic AGB Evolution
}

\author{
J. WAGENHUBER \\ Max-Planck-Institut für Astrophysik, Garching, Germany
}

As shown by Groenewegen \& de Jong (1993, $A \& A, 267,410)$, detailed knowledge of the secular luminosity variations of stars on the thermal pulsing (TP) AGB is an important ingredient for synthetic AGB evolution which enables one to study, apart from other ill-known quantities, the influence of different mass-loss descriptions by producing statistical data that can be compared to observations. This will not be possible by direct stellar evolution calculations for quite some time. Data from 38 AGB sequences (partly with OPAL opacities, nuclear network etc.), comprising three metallicities $\left(Z=0.02,0.008\right.$ and $\left.10^{-4}\right)$ and initial masses $M_{*} / M_{\odot}$ from 0.8 to 7 , all starting from the ZAMS and with a very high resolution, have been used to derive fit formulae on a statistical basis of more than 700 TPs. Except for hot-bottom burning, most of the quantities appear to be quite independent of the model physics. The dependence on $Z$ has been taken into account as well as "turn-on" effects which influence the behavior of the first 5-10 TPs of every sequence (this is crucial for low-mass stars which probably do not experience more). The formulae allow reconstructing the slow variations of $L_{*}$ between TPs and modeling the height and shape of the short-term peaks in order to study, for example, their influence if a threshold behavior of the mass loss is assumed, or objects which show rapid period changes presumably due to luminosity variations after a TP (Mattei, this volume).

As an example, the maximum luminosity $L_{\mathrm{A}}$ attained during quiescent hydrogen burning is given by a linear asymptotic relation, a correction which decreases with increasing $\Delta M_{\mathrm{c}} \equiv M_{\mathrm{c}}-M_{\mathrm{c}, 0}\left(M_{\mathrm{c}}\right.$ is the actual mass of the hydrogen-exhausted core, $M_{\mathrm{c}, 0}$ the core mass at the first TP), and a correction for hot-bottom-burning which is the only term that depends on the model, especially on the value of the mixing-length parameter, and the mass of the convective mantle $M_{\mathrm{m}} \equiv M_{*}-M_{\mathrm{c}}$ (in solar units):

$$
\begin{array}{r}
L_{\mathrm{A}}=\left(50900+5000 \lg \frac{Z}{0.02}\right)\left(M_{\mathrm{c}}-0.469\right)-10^{2.31+1.56 M_{\mathrm{c}, 0}+\left(39.5-115 M_{\mathrm{c}, 0}\right) \Delta M_{\mathrm{c}}} \\
+10^{1.38-0.33 \lg \frac{Z}{0.02}+\left(0.411+1.20 \Delta M_{\mathrm{c}}\right) M_{\mathrm{m}}} .
\end{array}
$$

